\begin{tabular}{|c|c|c|c|c|c|c|}
\hline \multirow{4}{*}{ Impact Factor: } & ISRA (India) & $=3.117$ & SIS (USA) & $=0.912$ & ICV (Poland) & $=6.630$ \\
\hline & ISI (Dubai, UAI & $=0.829$ & РИНЦ (Russia) & $=0.156$ & PIF (India) & $=1.940$ \\
\hline & GIF (Australia) & $=0.564$ & ESJI (KZ) & $=8.716$ & IBI (India) & $=4.260$ \\
\hline & JIF & $=1.500$ & SJIF (Morocco & $=5.667$ & OAJI (USA) & $=0.350$ \\
\hline
\end{tabular}

\section{SOI: $1.1 /$ TAS DOI: $10.15863 /$ TAS International Scientific Journal Theoretical \& Applied Science}

p-ISSN: 2308-4944 (print) e-ISSN: 2409-0085 (online)

Year: $2019 \quad$ Issue: $05 \quad$ Volume: 73

Published: $27.05 .2019 \quad$ http://T-Science.org

SECTION 4. Computer science, computer engineering and automation.
QR - Issue

QR - Article
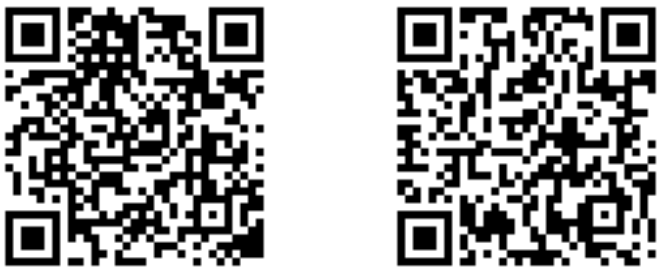

Valentin Nikolayevich Kiselev

Student,

Peter the Great St.Petersburg Polytechnic University mrexox@yahoo.com

Vadim Andreevich Kozhevnikov Senior Lecturer,

Peter the Great St.Petersburg Polytechnic University vadim.kozhevnikov@gmail.com

\title{
DEVELOPMENT OF THE AUTOMATIC SYNCHRONIZATION SERVICE FOR PACKAGES AND FILES FOR RPM AND DEB BASED DISTRIBUTIONS
}

Abstract: The article describes the approach to development of the system with a few independent services that handle synchronization of packages and files in RPM and DEB based distributions.

Key words: synchronization, Ruby, Rhel, CentOS, Debian, RPM, DEB, inotify, DRb, RPC, TOML.

Language: English

Citation: Kiselev, V. N., \& Kozhevnikov, V. A. (2019). Development of the automatic synchronization service for packages and files for RPM and DEB based distributions.. ISJ Theoretical \& Applied Science, 05 (73), $358-362$.

Soi: http://s-o-i.org/1.1/TAS-05-73-52 Doi: crossef https://dx.doi.org/10.15863/TAS.2019.05.73.52

\section{Introduction}

The synchronization problem of two and more computer systems appears when there is a need to keep them all in fresh state. Cluster systems use sharing resources and distributed file systems [1], kernel utilities to create something like RAID 1 on the systems via internet connection and other utilities of controlling the system resources and rescuing machines.

The main reason why these technologies appeared was the need to apply changes on a group of machines to make them act like a cluster. We miss something after all: configuration and program files updates. There is always a chance that something can change in requirements and all systems will need the update. That is why it is important for an administrator to think about synchronization of files and packages.

\section{Motivation}

The main disadvantages of existing state synchronization approaches are: master-slave architecture and manual actions. Configuration of these systems (e.g. Chef, Puppet, and Ansible) makes the administrator to create special files and apply them from a server to manually selected clients. It is handy for executing some commands but the whole process takes too much time and leaves a place for mistakes.

If there was a system that handles changes on one machine and sends them to another machines automatically, it would decrease a chance to mistaken and make synchronization more simple.

\section{Requirements}

The system must meet following requirements:

- Automatic updates of RPM and DEB packages;

- Automatic updates of selected files when they change;

- Decentralized synchronization.

These requirements lead to some restriction and features:

- The program works in a background;

- The program has a configuration file;

- The program works only in local network.

Technology review

Background processing in Linux is implemented via daemons controlled by initialization system. In almost all Linux distributions for now this initialization system is called Systemd [2]. 


\begin{tabular}{|c|c|c|c|c|c|c|}
\hline \multirow{4}{*}{ Impact Factor: } & ISRA (India) & $=3.117$ & SIS (USA) & $=0.912$ & ICV (Poland) & $=6.630$ \\
\hline & ISI (Dubai, UAE & $=0.829$ & РИНЦ (Russia & $=0.156$ & PIF (India) & $=1.940$ \\
\hline & GIF (Australia) & $=0.564$ & ESJI (KZ) & $=8.716$ & IBI (India) & $=4.260$ \\
\hline & JIF & $=1.500$ & SJIF (Morocce & $=5.667$ & OAJI (USA) & $=0.350$ \\
\hline
\end{tabular}

Most daemons get their configuration from special configuration files, kept under /etc [3]. Configuration file format vary from simple to complex, but the base are: INI, YAML, TOML, JSON, XML. TOML format is the most rich and readable if choosing between them. It has the following advantages:

- inclusive settings (INI doesn't have them),

- insensitive to spaces and tags (YAML is not),

- provides understandable syntax with comments (not like JSON);

Hierarchical structure allows to describe configuration of many services in one file.

There are also unique configuration file formats for such programs as cron, nginx, freeradius, ntp, etc.

Discovering systems in the network needs using transport layer UDP protocol and broadcast requests. This technology is widely used in many protocols that need to discover some server in a local network, for instance DHCP, DNS.

The synchronization system is decentralized and that is why every node of network has the whole database of events. When any node goes offline and then recovers, it asks for events and filters them to apply only new. The redundancy of data make the whole system more resistant to unfortunate occasions.

To start with, it is important to choose a programming language for the realization. There are requirements to a programming language:

- ability to be extended fast and easy,

- ability to change realization of any module not touching others,
- RPC solution out-of-the-box,

- lots of stable packages and extensions.

The only type of programming languages that fits these requirements is scripting programming language. The choice of Ruby is more appropriate, because it has an RPC solution from the standard library called DRb. Also Ruby provides object oriented design and fits into event-driven design by providing blocks - code, that can be passed to a function and yielded in it, if needed.

As it was mentioned, there are some well-known approaches to node discovering. The design is very simple: a server listens on a specific port, and the client requests sends the requests on that port. There are three ways to do it in Ruby:

- UDP socket,

- Event Machine,

- SSDP.

Every approach encapsulate sending a UDP broadcast requests but provides different mechanisms and integrations.

SSDP - is a network protocol for discovering network services. It is a common standard for service discovering but may look overabundant. This protocol is the standard for network discovery and it is the main advantage of using it.

UDP socket usage makes the programmer to perform some actions in right order. For example, sending a broadcast message using UDP socket includes:

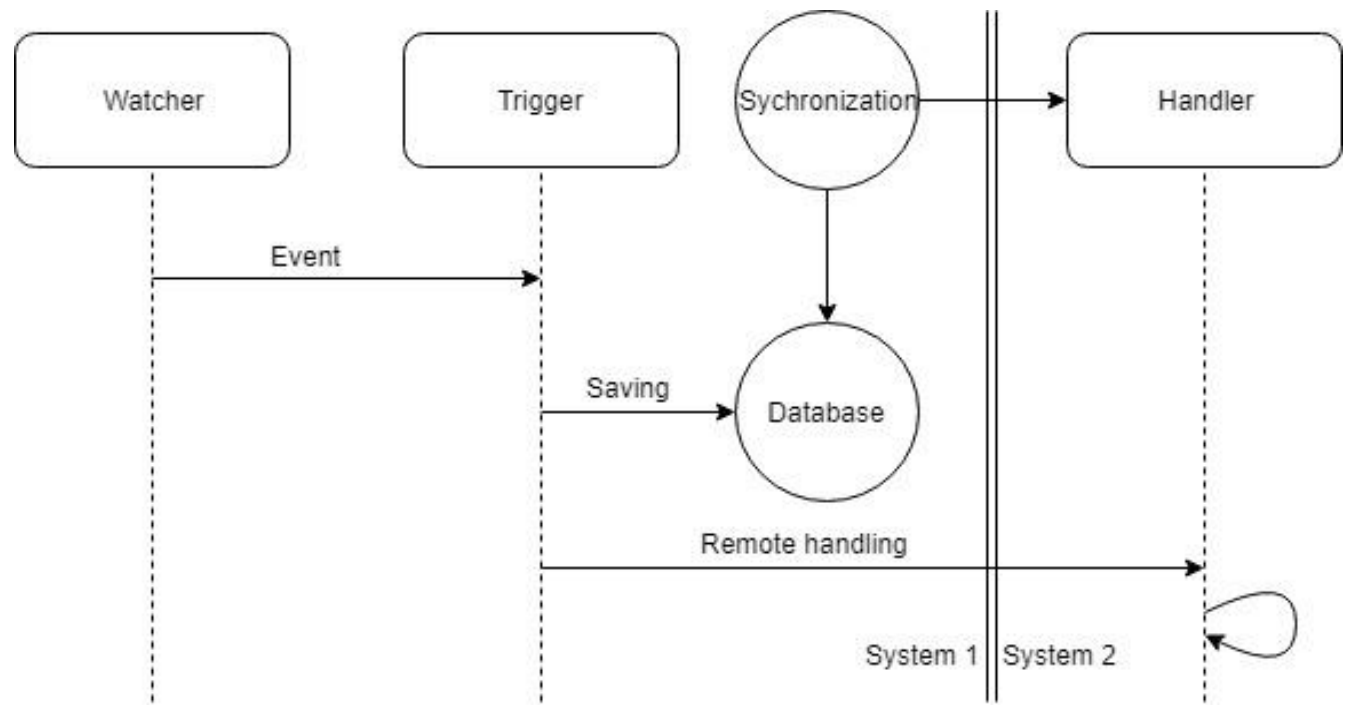

Figure 1 - Behavior of the monitoring system port;

- Opening a UDP socket on special host and

- Setting up the socket to be broadcast;

- Sending the broadcast message;

- Closing the socket.
The server side part is:

- Opening a UDP socket on 0.0.0.0 to accept all requests;

- Handling incoming requests. 


\begin{tabular}{|c|c|c|c|c|c|c|}
\hline \multirow{4}{*}{ Impact Factor: } & ISRA (India) & $=3.117$ & SIS (USA) & $=0.912$ & ICV (Poland) & $=6.630$ \\
\hline & ISI (Dubai, UAE & $=0.829$ & РИНЦ (Russia & $=0.156$ & PIF (India) & $=1.940$ \\
\hline & GIF (Australia) & $=0.564$ & ESJI (KZ) & $=8.716$ & IBI (India) & $=4.260$ \\
\hline & JIF & $=1.500$ & SJIF (Morocce & $=5.667$ & OAJI (USA) & $=0.350$ \\
\hline
\end{tabular}

Setting up Event Machine with UDP server is a bit harder. Using Event Machine is valuable only when using it as the base for all services. Also using it for RPC provides a multi-lingual solution [4], so new services may be written for such system in another programming language.

There are some well-known RPC approaches for Ruby:

- JSON-RPC,

- DRb,

- SOAP,

- XML-RPC,

- Apache Thrift.

$\mathrm{DRb}$ is the most native approach and provides out of the box solution for remote procedure calls [5]. Anyway, the realization of communication may be changed because of modular architecture of the program.

\section{Architecture}

Any program can be described with two characteristics. The first one is its architecture. Good architecture decreases the cost of further changes and allows to widen features with low cost. The second characteristic is the behavior. As soon as program can manage well some critical states its behavior is more reliable. The aim is to reach good architecture and suppose correct behavior of the program [6].

Object oriented approach was used to provide independent modules so the SOLID principles could be reached [7]. The program consists of four microservices, four daemons that run independently:

- evemond - provides monitoring and notifying about events like package installation or file change;

- evehand - provides business logic, a reaction on events happening in the system;

- evesyncd - provides synchronization between nodes;

- evedatad - provides database connection and saves all events to database.

The whole system works correctly only when all the daemons run. Each action of monitoring, handling, saving to database and synchronization are implemented in separate modules.

The interactions between modules are rather simple (pic. 1). The event formed in the Watcher module is catched by the Trigger module and saved via Database daemon. Then the event goes to other systems and gets handled by the Handler module, which applies the change and saves it to local database too.

Watcher module implements monitoring changes and adding events to an event queue. This module is a factory for two main watching modules: packages watcher and file watcher. The event queue is processed by Trigger module in the same daemon but different thread.
Trigger module is a controller, which handles all actions a system, must perform to save, apply and share an event. The main task of this module is to control the flow of events from Watcher module. In addition, when an event is received from Handler module, it means the remote node has sent the event. That is why after applying an event and receiving it again from local Watcher module the Trigger must ignore this event. The purpose of ignoring is not to duplicate messages in local network. Duplicating one message about package upgrade by hundreds of nodes may cause decreasing of throughput.

Reacting logic is in the Handler module. This module implements the service that handles events from remote nodes. With the synchronization service, they make a public interface of the system for interactions.

Inter-process communication is implemented in an IPC module. It provides server and client side. Using $\mathrm{DRb}$ technology makes it easy to call corresponding methods directly without wrapping in any communication protocol. In addition, DRb provides message encryption using SSL certificates.

Besides mechanisms for calling external methods this module provides a special type of objects to be used as messages. Package and file events are wrapped in a special class with metadata that can be marshalled into the text and saved into the database. After extracting it from the database, it can be easily parsed into a usual Ruby object. Metadata for these events includes a timestamp and actual object name that are used as a unique key for a particular event.

Using standard Marshall Ruby module does not fit the requirements for two reasons. The first one marshalled objects will not be able to be loaded via another language. This is important because there must be a place of widening the system with other utilities [7] and they may be written in other programming languages, for instance $\mathrm{C}++$, and there must be a way to implement an event class and parse it in any language. Therefore, the second reason is that Marshall module provides marshalling into a binary format, not textual. Binary format is not so easy to send within network, that is why textual format was chosen.

Database class implements working with a database. For this project, the LMDB database fitted the best. There were following reasons:

- No reasons to use relational database, because there are no relations;

- LMDB is called the fastest key-value database [8];

- LMDB is not client-server database but provides transactions using special locks;

- LMDB supports parallel reading;

- It works "out of the box" installed from Ruby gem.

LMDB stores the data in files of a specified folder. So, the replication can be easily made by 


\begin{tabular}{|c|c|c|c|c|c|c|}
\hline \multirow{4}{*}{ Impact Factor: } & ISRA (India) & $=3.117$ & SIS (USA) & $=0.912$ & ICV (Poland) & $=6.630$ \\
\hline & ISI (Dubai, UAE & $=0.829$ & РИНЦ (Russia & $=0.156$ & PIF (India) & $=1.940$ \\
\hline & GIF (Australia) & $=0.564$ & ESJI (KZ) & $=8.716$ & IBI (India) & $=4.260$ \\
\hline & JIF & $=1.500$ & SJIF (Morocce & $=5.667$ & OAJI (USA) & $=0.350$ \\
\hline
\end{tabular}

copying its files. This approach is useful if we would like to implement offline synchronization, loading and applying events from a database file.

Sync class provides synchronization between nodes and controls discovering of systems. Discovering is implemented via UDP server that listens to the "world" and UDP broadcast requests that are sent seldom. When the system starts, the request is sent automatically and all nodes that answers are asked about events. After that timestamps are analyzed and compared with saved events on the local machine. When the algorithm finds the missing events the synchronization service requests them from corresponding nodes and applies changes to the system. After that, the node becomes synchronized with the others.

\section{Behavior}

The behavior demonstrates business logic and processes in the system. Every service has its own responsibility and plays its role. That is why the system improvement and error checking is not as complicated as it could be if it was monolithic.

Evemond daemon initializes Trigger and Watcher modules and starts monitor-react-handle event cycle. It also starts an IPC server so other threads and services can call its public methods to control the watching behavior.

Evehand daemon starts public IPC server so other daemons and nodes can interact with it. Evehand processes following requests:

- request for getting events timestamp list from local database,

- request for getting particular events in marshalled form, system.

- request for handling events from other

Evehand communicates with the watcher daemon. For example, when evehand daemon handles request to update some package, the watcher service notifies about package event. The handler's job is to say Trigger module to ignore particular packages and not to perform any usual action.

Evedatad daemon is used for saving events and providing data from the database (fig. 1 and 2).

Evesync daemon is responsible for synchronization. To perform synchronization it needs to send some messages to remote nodes, ask for missed events and apply them using handler service (pic. 2).

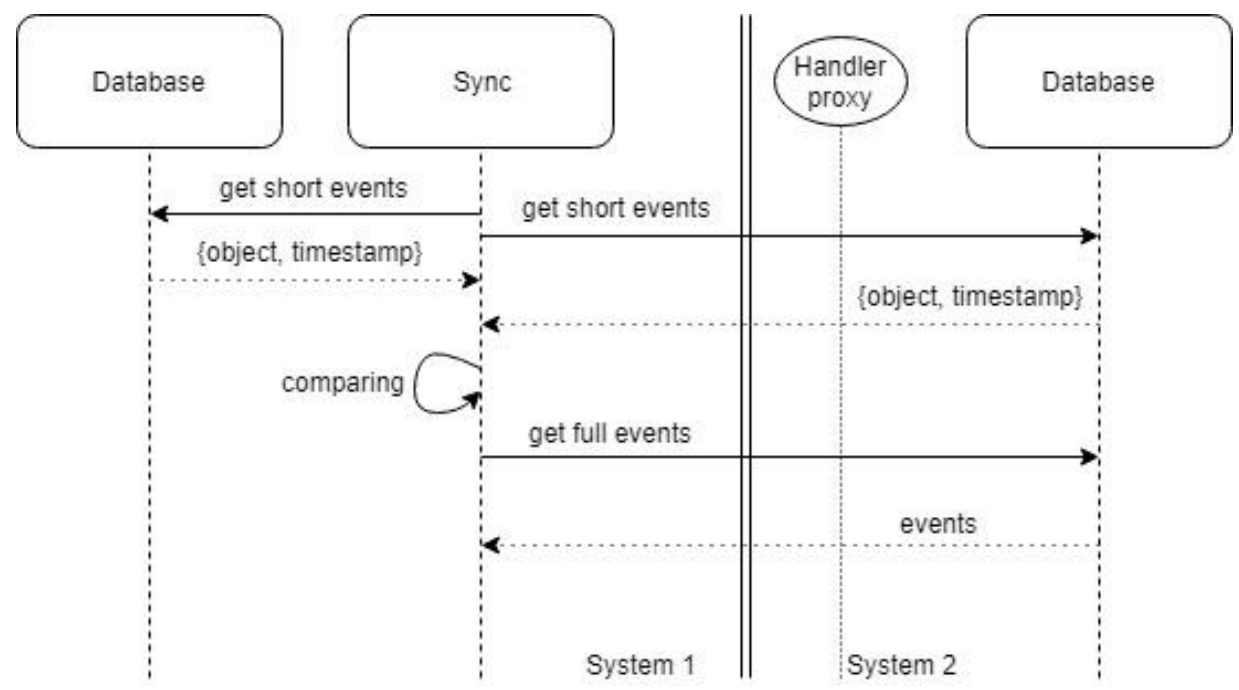

Figure 2 - Synchronizing events.

To provide working with RPM and DEB packages every time the system starts it parses /etc/osrelease file to find out what distribution it was started on. When this file contains words like "rhel" or "centos", it means that rpm packages and yum package manager might be used. When this file contains the word "debian", it means that apt package manager and "dpkg" package control utility are used.

To catch changes of package system the monitor lists all packages in the system and saves names and versions once the specified interval. On the next iteration, it compares this snapshot with the previous one and finds out the changes. These changes become package events that are catched in the Trigger module.

The same way file changes work. When the system starts, files that should be monitored are watched by inotify subsystem. Files Watcher module gather all events for a small period and parses them into the appropriate event objects that the Trigger module catches. 


\begin{tabular}{|c|c|c|c|c|c|c|}
\hline \multirow{4}{*}{ Impact Factor: } & ISRA (India) & $=3.117$ & SIS (USA) & $=0.912$ & ICV (Poland) & $=6.630$ \\
\hline & ISI (Dubai, UAE & $=0.829$ & РИНЦ (Russia & $=0.156$ & PIF (India) & $=1.940$ \\
\hline & GIF (Australia) & $=0.564$ & ESJI (KZ) & $=8.716$ & IBI (India) & $=4.260$ \\
\hline & JIF & $=1.500$ & SJIF (Morocce & $=5.667$ & OAJI (USA) & $=0.350$ \\
\hline
\end{tabular}

Testing

There is a testing framework for Ruby programming language called Rspec. This framework makes possible testing algorithms of synchronization and database service API. This is the main point for using unit tests in this project. In addition, Rspec allows freezing some business logic and making sure that it will not change its behavior in some time [9].

Testing business logic is more complicated. Container-based approached was developed to make it easier [10]. The Docker container is used to install all the dependencies on a clean system. Two and more replicas get started for testing using docker-compose program. Docker-compose provides a utility that allows uniting many instances of container into one network. It allows using containers as standalone virtual machines in a local network. In addition, there is a possibility to mount project root into every container file system. Therefore, all changes in a project appear in all containers. It allows automatically update the code that runs in the containers.
When an event occurs on any container it is automatically synchronized between other containers and we can check it via logs or commands of package manager.

Using containers for testing is cheaper and faster than using virtual machines. It is more appropriate because every time a container runs it has a clean system.

\section{Conclusion}

To implement an automatic synchronization between computers with one Linux distribution there were four services developed and tested. The system provides monitoring, applying, saving and synchronizing events. Micro-service architecture was chosen, because it makes development and widening easier and does not bind API between independent elements of the system.

Using unit testing allowed making sure that new features will not damage old algorithms. Using Docker containers made testing synchronization easier also.

\section{References:}

1. (n.d.). HDFS, Bauman National Library. Retrieved May 10, 2019, from https://ru.bmstu.wiki/HDFS_(Hadoop_Distribut ed_Filesystem)

2. (n.d.). Systemd, Wikipedia. Retrieved May 14, 2019, from https://ru.wikipedia.org/wiki/Systemd

3. (n.d.). Filesystem Hierarchy Standard, Wikipedia. Retrieved May 14, 2019, from https://en.wikipedia.org/wiki/Filesystem_Hierar chy_Standard

4. (n.d.). EventMachine UDP Server Example, Parrotty Blog. Retrieved May 15, 2019, from https://parroty00.wordpress.com/2013/07/14/ev entmachine-udp-server-example/

5. (n.d.). DRuby aka DRb - the baseline for distributed systems on Ruby. Principles of working, Habrahabr. Retrieved May 16, 2019, from: https://habr.com/ru/post/143671/
6. Sandi, M. (2018). Practical Object-Oriented Design: An Agile Primer Using Ruby (2nd Edition), Addison-Wesley Professional, p.288.

7. Robert, C. M. (2017). Clean Architecture: A Craftsman's Guide to Software Structure and Design. Prentice Hall, p.432.

8. (n.d.). Lightning Memory Mapped Database, Wikipedia. Retrieved May 15, 2019, from https://ru.bmstu.wiki/LMDB_(Lightning_Mem ory-Mapped_Database)

9. (n.d.). Introduction to Ruby and RSpec, Medium. Retrieved May 19, 2019, from https://medium.com/craftacademy/introduction-to-ruby-and-rspec$135 \mathrm{da} 4051802$

10. (n.d.). Manuel Weiss. How Docker Makes Testing More Efficient. Retrieved May 19, 2019, from: https://blog.codeship.com/testing-withdocker/ 